\title{
A Patient with Recurrent Hypokalaemia
}

\author{
CS BALA $^{\mathrm{a}}$, I REZA $^{\mathrm{b}}$, MABI MOMEN ${ }^{\mathrm{c}}$, HAMN AHASAN $^{\mathrm{d}}$
}

(J Bangladesh Coll Phys Surg 2016; 34: 228-230)

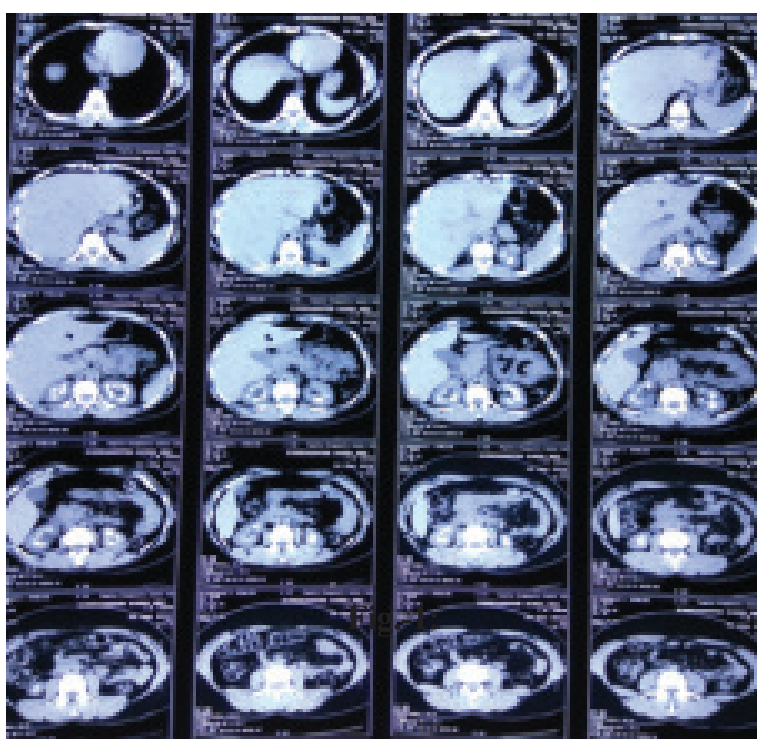

This is the CT Scan of abdomen of a patient who is 37 year-old lady presented with repeated episodes of high grade fever with chills and rigor, increase frequency and burning sensation during micturation for one year. She noticed frequent passage of small stone like particles for the last 3 months. She had profound generalized weakness. She had history of similar episodes of disease and hospital admission one year back.

On physical examination She was febrile and was so weak to move her limbs. She had no history of joint

a. Dr. Chandra Shekhar Bala, Junior Consultant (Medicine), NINS and Hospital.

b. Dr Ishrat Reza, Registrar, Medicine, Popular Medical College, Dhaka.

c. Dr. Md A Basit Ibne Momen, Asstt Registrar(Medicine).

d. Prof. H.A.M. Nazmul Ahasan, Professor of Medicine, Popular Medical College, Dhaka.

Address of Correspondence: Dr. Chandra Shekhar Bala, Junior Consultant (Medicine), NINS and Hospital. pain, dryness of mouth or grittiness of eye. She was not on any medication. Her urine analysis revealed plenty of pus cell with few RBC but Urine Culture and sensitivity could not reveal growth of any organism. Neutrophilic leukocytosis was noted in full blood count. Renal function test within normal limit. Her Potassium was $1.9 \mathrm{mmol} / \mathrm{Liter}$ with raised Chloride $(124 \mathrm{mmol} / \mathrm{L})$ level and decreased bicarbonate level $(21 \mathrm{mmol} / \mathrm{L}$. Her Venous blood $\mathrm{P}^{\mathrm{H}}$ was within 7.3. Her Urinary Potassium excretion was high (74.78 mmol/24 hours). Her serum calcium, Phosphate and serum PTH were normal within limit..

Plain X-ray KUB region showed bilateral renal calcification. USG of whole abdomen showed multiple calculi mostly periphery of both kidneys. Ultrasonography could not opine conclusively whether it was multiple large calculi or medullary calcifications of both kidneys.

CT scan of abdomen showed symmetrical medullary calcification of both kidney. Renal parenchyma shows 

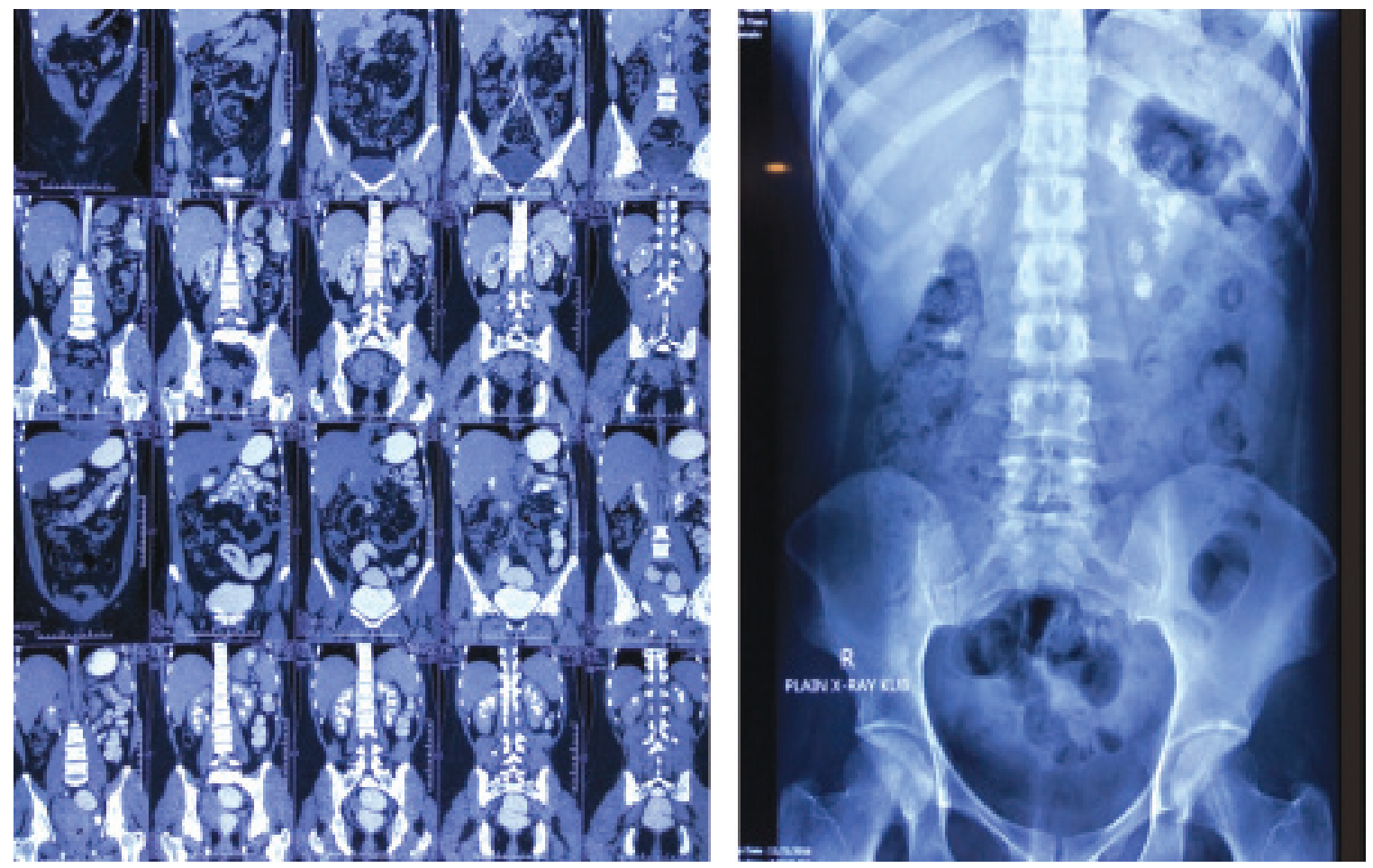

Fig.-2: CT abdomen sagital view(on the left) and X-ray KUB (on the right) of the same patient.

normal width with normal size, shape and position of kidneys. Pelvicaliceal sytem is not dilated and have no calculus or mass lesion.

Acid load test revealed persistently raised urinary $\mathrm{p}^{\mathrm{H}}$ (above 6.8) in the following eight hours after intake of ammonium chloride $(0.1 \mathrm{mg} / \mathrm{Kg}$ body weight). In normal individual it would have fall below 5.2 between 2 nd and 5 th hour after ingestion of ammonium chloride. This is consistent with renal tubular acidosis. The final diagnosis was Distal Renal Tubular Acidosis with Nephrocalcinosis with recurrent Urinary tract Infection.

\section{Discussion:}

Nephrocalcinosis is associated with generalized increase in the concentration of calcium and the deposition of calcium salts in the renal parenchyma ${ }^{1}$. The most common causes of this condition are primary hyperparathyroidism, renal tubular acidosis, medullary sponge kidney, idiopathic hypercalciuria, hyperoxaluria (primary, enteric or toxic), secondary hypercalcemia (sarcoidosis, neoplasm and osteoporosis) and drugs (including furosemide, acetazolamide and amphotericin B). This condition has no clinical symptoms in the early phase, except in cases of nephrolithiasis (kidney stone) and ureteric colic. The condition can progress to chronic kidney failure, and the prognosis depends on the underlying cause. Laboratory examinations may reveal microscopic pyuria even in the absence of urinary infection. This condition differs from kidney stones by the formation of calculi in the excretory tract. The increase in calcium content in the kidneys occurs in three phases (chemical, microscopic and macroscopic) with different degrees of kidney damage. Macroscopic nephrocalcinosis is classified as cortical (3\% of cases), medullary ( $97 \%$ to $98 \%$ of cases) or mixed (involving the renal cortex and medulla). ${ }^{2}$

Nephrocalcinosis can be detected using imaging examinations such as radiography, ultrasound or computed tomography. Axial computed tomography without contrast is considered adequate for differentiating cortical and medullary nephrocalcinosis and demonstrating the relationship between calcifications in the parenchyma and the calyceal system, allowing the precise localization of the calcifications.

Nephrocalcinosis with low serum potassium is shrinks the differentials to few conditions like Bartter's 
syndrome, Liddle syndrome, Primary hyperadrenalism and Distal Renal Tubular Acidosis(dRTA). ${ }^{3}$ This patient has acidosis and increased urinary potassium excretion. Acid load test revealed inability to acidify urine following ammonium chloride intake. Thus we reached the final diagnosis of Distal Renal Tubular Acidosis(dRTA). We excluded other possible cause of nephrocalcinosis.

Distal RTA(RTA type I) is a rare disorder characterized by non-anion gap hyperchloremic acidosis and hypokalemia. In this condition, the alpha intercalated cells of the cortical collecting duct of the distal nephron fail to secrete acid into the urine. This failure of acid secretion leads to an inability to acidify the urine to a $\mathrm{pH}<5.5$. Because renal excretion is the primary means of eliminating acid from the body, there is consequently a tendency towards systemic acidemia. This leads to the clinical features of RTA type I, which include: Normal anion gap hyperchloremic metabolic acidosis; Hypokalemia (from multiple mechanisms, but often severe during periods of stress); Nephrocalcinosis; Nephrolithiasis (related to an inability to acidify urine); Hypercalciuria, and low urinary citrate); Loss of calcium from bones (which can cause rickets in children and osteomalacia in adults). ${ }^{4}$ The clinical manifestations of RTA type I depend upon the disease type and severity of the disease. Brenner et al. indicated that type I RTA occurred more frequently in patients older than 18 years of age and had a female predominance. ${ }^{5}$ Hypokalemia probably results from increased kaliuresis due to renal tubular leakage, decreased proximal tubular reabsorption in the face of acidosis and hypocapnia, and aldosterone stimulation. ${ }^{6}$ Hypercalciuria, hyperphosphaturia, hypocitraturia and a high urinary $\mathrm{pH}$ are the main events that predispose RTA patients to develop renal stones. Urinary citrate is an inhibitor of the crystal aggregation and precipitation. When citrate excretion is reduced, more calcium is chelated, thus aggravating urolithiasis and nephrocalcinosis. ${ }^{7,8}$

\section{References:}

1. Monk RD, Bushinsky DA. Nephrolithiasis and nephrocalcinosis. Johnson RJ, Feehally J, eds. Comprehensive Clinical Nephrology. 2nd ed. Mosby; 2003. $731-4$

2. Tasic V, Gucev Z. Nephrolithiasis and Nephrocalcinosis in Children - Metabolic and Genetic Factors. Pediatr Endocrinol Rev. 2015 Sep. 13 (1):468-76.

3. Evan AP, Lingeman J, Coe F, et al. Renal histopathology of stone-forming patients with distal renal tubular acidosis. Kidney Int. 2007 Apr. 71(8):795-801

4. Batlle D, Moorthi KM, Schlueter W, Kurtzman N. Distal renal tubular acidosis and the potassium enigma. Semin Nephrol 2006 Nov;26(6):471- 478.

5. Brenner RJ, Spring DB, Sebastian A, McSherry EM, Genant HK, Palubinskas AJ, et al. Incidence of radiographically evident bone disease, nephrocalcinosis, and nephrolithiasis in various types of renal tubular acidosis. N Engl J Med 1982 Jul;307(4):217-221

6. Blake-Palmer KG, Karet FE. Cellular physiology of the renal H+ATPase. Curr Opin Nephrol Hypertens 2009 Sep;18(5):433-438

7. Rothstein M, Obialo C, Hruska KA. Renal tubular acidosis. Endocrinol Metab Clin North Am 1990 Dec;19(4):869887.

8. Simpson DP. Citrate excretion: a window on renal metabolism. Am J Physiol 1983 Mar;244(3):F223-F234. 